Quantitative vibro-acoustography of tissue-like objects by measurement of resonant modes

This content has been downloaded from IOPscience. Please scroll down to see the full text.

2017 Phys. Med. Biol. 62107

(http://iopscience.iop.org/0031-9155/62/1/107)

View the table of contents for this issue, or go to the journal homepage for more

Download details:

IP Address: 14.139.128.21

This content was downloaded on 07/02/2017 at 04:57

Please note that terms and conditions apply.

You may also be interested in:

Simulations of radiation force induced vibrations on a blood vessel wall

Janne Heikkilä, Tero Karjalainen, Marko Vauhkonen et al.

Probing the dynamics of tissue with radiation force

Mostafa Fatemi and James F Greenleaf

Viscoelastic characterization of thin tissues

Bojan B Guzina, Kairat Tuleubekov, Dalong Liu et al.

Quantitative viscoelastic parameters measured by harmonic motion imaging

Jonathan Vappou, Caroline Maleke and Elisa E Konofagou

Simultaneous identification of elastic properties, thickness, and diameter of arteries excited with ultrasound radiation force

Parikshit Dutta, Matthew W Urban, Olivier P Le Maître et al.

Multi-parametric monitoring and assessment of high-intensity focused ultrasound (HIFU) boiling by harmonic motion imaging for focused ultrasound (HMIFU): an ex vivo feasibility study

Gary Y Hou, Fabrice Marquet, Shutao Wang et al.

Quantitative sonoelastography for the in vivo assessment of skeletal muscle viscoelasticity

Kenneth Hoyt, Timothy Kneezel, Benjamin Castaneda et al.

Tissue resonant acoustic spectroscopy using magnetomotive nanotransducers in OCT

Amy L Oldenburg and Stephen A Boppart 


\title{
Quantitative vibro-acoustography of tissue-like objects by measurement of resonant modes
}

\author{
Dibbyan Mazumder ${ }^{1}$, Sharath Umesh ${ }^{1}$, Ram Mohan Vasu ${ }^{1}$, \\ Debasish Roy ${ }^{2,4}$, Rajan Kanhirodan ${ }^{3}$ and Sundarrajan Asokan ${ }^{1}$
}

1 Department of Instrumentation \& Applied Physics, Indian Institute of Science, Bangalore 560 012, India

${ }^{2}$ Computational Mechanics Lab, Civil Engineering, Indian Institute of Science, Bangalore 560 012, India

3 Department of Physics, Indian Institute of Science, Bangalore 560 012, India

E-mail: dibbyan@gmail.com, sharath.shs@gmail.com, vasu@iap.iisc.ernet.in, royd@civil.iisc.ernet.in, rajan@physics.iisc.ernet.in and sasokan@iap.iisc.ernet.in

Received 8 August 2016, revised 4 October 2016

Accepted for publication 25 October 2016

Published 13 December 2016

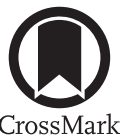

\begin{abstract}
We demonstrate a simple and computationally efficient method to recover the shear modulus pertaining to the focal volume of an ultrasound transducer from the measured vibro-acoustic spectral peaks. A model that explains the transport of local deformation information with the acoustic wave acting as a carrier is put forth. It is also shown that the peaks correspond to the natural frequencies of vibration of the focal volume, which may be readily computed by solving an eigenvalue problem associated with the vibrating region. Having measured the first natural frequency with a fibre Bragg grating sensor, and armed with an expedient means of computing the same, we demonstrate a simple procedure, based on the method of bisection, to recover the average shear modulus of the object in the ultrasound focal volume. We demonstrate this recovery for four homogeneous agarose slabs of different stiffness and verify the accuracy of the recovery using independent rheometer-based measurements. Extension of the method to anisotropic samples through the measurement of a more complete set of resonant modes and the recovery of an elasticity tensor distribution, as is done in resonant ultrasound spectroscopy, is suggested.
\end{abstract}

Keywords: biomedical imaging, elastic recovery, image reconstruction, inverse problem, ultrasonic imaging

(Some figures may appear in colour only in the online journal)

\footnotetext{
${ }^{4}$ Author to whom any correspondence should be addressed.
} 


\section{Introduction}

Non-invasive measurements of the optical and mechanical properties of soft-tissue organs have provided valuable data with which physicians can diagnose disease and assess its progression, as well as verify the efficacy of treatment procedures. The most prominent among pathologies is cancer in soft-tissue organs, and one of the properties that can monitor its growth is the shear modulus. The experimental measurement of any visco-elastic parameter of an object requires the assessment of the displacement introduced through the application of a known force. These methods of mapping elasticity, or a related quantity, namely, the strain field, have given rise to the area of study known as elastography. In one of the methods of implementing elastography, called dynamic elastography, which is of relevance to the work here, a time harmonic excitation leads to a time harmonic displacement which is measured ( $\mathrm{Ji}$ and McLaughlin 2003). This approach is also used to excite and measure the object's elastic resonance frequencies. From the measurement of these frequencies characterizing the free vibration response, the specimen's elastic properties, density and shape can be assessed (Mitri et al 2003, Huber et al 2006, Bernal et al 2011, Fan et al 2013). By fitting the measured resonance frequencies with a model to compute them, the elastic properties of both isotropic and anisotropic rock samples have been recovered by Zadler et al (2004) and Migliori et al (1993), as have the elastic constants of human dentin by Kinney et al (2004). Location-specific harmonic excitation is applied by a focused ultrasound beam, and the vibration amplitude is measured at certain selected points on the object's surface. When the dissipation in the object is not high, many resonant modes are easily measured, from which a robust recovery of the elastic constants is demonstrated using this method. This has come to be known as resonant ultrasound spectroscopy (RUS) (Visscher et al 1991, Migliori et al 1993, Kinney et al 2004, Zadler et al 2004).

Localized harmonic excitation with a focused ultrasound beam (also called remote palpation) is not new in the elasticity imaging of tissues (Konofagou et al 2003, Heikkilä et al 2010). Through proper beam forming, two ultrasound beams of slightly different frequencies are made to intersect in a region of interest (ROI) where a localized sinusoidal force at the frequency difference can be applied. The local sinusoidal forcing, it is shown, creates a local oscillatory strain confined only to the vicinity of the ROI (Fatemi and Greenleaf 1999). However, the ultrasound-induced force can generate a transverse shear wave propagating through the object creating a time varying displacement that can be tracked by a Doppler ultrasound scanner (Barannik et al 2002). Another probe used in remote palpation elastography is the ultrasound pulse echo correlator (Nightingale et al 2001). A major turning point in making the remote-palpation-based method useful is the discovery of a secondary acoustic emission (the longitudinal wave), called the vibro-acoustic emission, originating at the ROI owing to the local forcing and local vibration. The amplitude and phase of this acoustic beam, detected on the boundary of the object, is used to create a qualitative representation of the mechanical property of the ROI, which forms a 'pixel' of the cross-section of the object being scanned. By allowing the ROI to scout a cross-section of biological objects such as an excised human liver, kidney or prostate, images showing lesions with higher stiffness in terms of the detected low acoustic intensity are reported (Alizad et al 2007, Mitri et al 2008, Zhai et al 2010, Mitri and Kinnick 2012). In addition, successful attempts have also been reported to arrive at quantitative images of the shear modulus distribution of the medium surrounding the ROI, from the measured spatial distribution of dynamic displacement history as the shear wave traverses through the object (Zhai et al 2010). On the other hand, to produce a quantitative image by raster scanning the object with the ROI, the effect of the inhomogeneous visco-elastic medium surrounding the ROI (as well as the water medium in which the entire object is immersed) 
on the acoustic field originating from the ROI has to be accounted for. Thus, as noted by Konofagou et al (2004), parameter estimation from vibro-acoustography (VA) (Brigham et al 2007) involves solving a difficult inverse problem. The associated difficulties notwithstanding, there have been successful attempts in the past to 'invert' data for the quantitative elasticity parameters of the ROI (Brigham et al 2007, Aguilo et al 2010).

Our objective here is the quantitative recovery of the elastic modulus from VA data. For measuring the vibro-acoustic pressure, we use a sensitive, wide-spectral band optical device, the fibre Bragg grating (FBG) (see appendix for details), and we measure only the VA frequencies at which the spectra peak. We claim, with proof through computation, that these frequencies are in fact the resonant (natural) frequencies of the ROI set in vibration by the locally applied force through focused ultrasound transducers. We also demonstrate that the shear displacement field produced as a consequence (referred to as an oscillatory movement by Fatemi and Greenleaf (1999)), though confined to the vicinity of the ROI, couples with the ultrasound-induced pressure waves therein, which, in turn, propagate to the boundary of the object, carrying information on the shear-elastic properties of the neighbourhood of the ROI. Because the 'source' of the propagating acoustic radiation is the vibrating region of the ROI, the natural frequencies of the vibrating ROI (VROI) so influence the propagating pressure field that the spectrum of the measured boundary pressure can be used to read out the spectrum of the VROI. A theoretical basis of this claim is established in section 2.2. Since these natural frequencies can be inferred from the VA spectrum (we measure only one dominant natural frequency in our experiments), one can employ a simple inversion procedure, such as the method of bisection used in this work, to recover the average elastic modulus of the ROI. We note that both natural frequencies and mode shapes, recovered from the measurements of vibrating structures, have routinely been employed to analyze such structures in the industry, in what has come to be known as experimental modal analysis (Schwarz and Richardson 1999, Kranjc et al 2016, Golinval). FBG sensors themselves were demonstrated by dos Santos et al (2015) for analysis of the structures for strain modal frequencies and shapes.

The summary of the rest of the paper is as follows. In section 2, we revisit vibro-acoustography, showing the dependence of the acoustic amplitude on the visco-elastic properties and density of the ROI. An elucidation on the relevant mechanics that enables an understanding of the transport of local shear-displacement information through the acoustic wave is provided. Here we also numerically study the variation of the resonant frequency of the vibrating region with the shear modulus of the material, represented in our case by the percentage of agarose used to mould the phantoms used in the experiments later. Section 3 describes the experiments: first the agarose phantom preparation, then the measurement of the VA spectrum through the FBG mounted on a sensitive cantilever. The peaks in the spectra are noted and compared with the theoretically derived resonant mode(s) of the vibrating region. Once the match is found satisfactory, in section 4, the measured spectral peak is used as a reference to adjust the computed resonant mode to match the measured VA peak, using the shear modulus as a parameter. Section 5 contains a discussion of the results and concluding remarks.

\section{Theory}

\subsection{Vibro-acoustography}

A sinusoidally varying low frequency radiation force at a specified location of the object, defined by the intersection of the focal volumes of two focusing ultrasound transducers, interacts with the material of the object at the location, resulting in a secondary acoustic wave source from the region. This acoustic wave, detected exterior to the body, contains information 
on the size, density and shear-elastic properties of the body pertaining to the intersecting volume. In VA, the amplitude and/or phase of this acoustic wave, emanating from a localized region of the body, is used either directly to construct an image representing its elastic properties, (Fatemi and Greenleaf 1998) or, after inversion, a true quantitative image (Brigham et al 2007, Aguilo et al 2010) by raster scanning the object with the ultrasound focal volume. A coupling of the equation for linear momentum balance, reflecting the shear-dominated localized deformation in the VROI, with that for the propagating acoustic (pressure) wave ensures that information on the shear deformation is encoded within the detected pressure signal, even though shear waves attenuate much faster (Madsen et al 1983). It is also reported that the conversion of the energy of vibration into an acoustic wave is affected by the mechanical admittance of the material of the ROI (Fatemi and Greenleaf 1998, 1999). An introduction to the concept (Fatemi and Greenleaf 1998), details of the theoretical framework (Fatemi and Greenleaf 1999), demonstration of its application in medical imaging (Alizad et al 2004, 2007, Mitri et al 2008, Zhai et al 2010, Mitri and Kinnick 2012) and quantitative visco-elastic property recovery (Brigham et al 2007, Aguilo et al 2010) are all available in a number of seminal papers from the group of Greenleaf and Fatemi. Before offering our explanation for VA by deriving an equation of propagation for the acoustic wave with a source term confined to the ROI, carrying information on the local shear displacement, density and shape of the region, it is pertinent to recall the main findings of their work. (1) The detected acoustic power spectrum is proportional to the square of the ultrasound input pressure, the vector drag coefficient of the object to incident ultrasound which represents the absorption and scattering coefficients of the object and the 'acoustic outflow', which is the result of the sinusoidal vibration of the ROI pressing against the background. (2) The effect of the intervening medium through which the acoustic wave travels to the detector on the acoustic spectrum is represented by a multiplicative frequency response of the medium. (3) Whereas the drag coefficient is affected by the projected area of the intersecting ultrasound beams on the object cross-section, the acoustic wave amplitude depends on the shape and area of the VROI projected. (4) The attenuation of high- and low-frequency acoustic waves, i.e. the input ultrasound beams which cause the forcing in the ROI and the secondary acoustic emission from it respectively, play an important role in the quality of images obtained from vibro-acoustography. For example, the absorption of ultrasound waves by the background object makes the point spread function of the imaging system ride on a pedestal, affecting the contrast of the recovered image (Fatemi and Greenleaf 1999). Since low-frequency compressional waves, which are what VA signals are, are absorbed very little by soft-tissue material, the SNR of the received acoustic emission is not greatly affected by the background tissue material. Moreover, inhomogeneous absorption variation in the background makes the pressure created at the ROI dependent on its location, thus affecting the true representation of the mechanical properties by the VA signal. (5) Quantitative recovery of material property from the signal is demonstrated by successfully solving an associated inverse problem (Brigham et al 2007, Aguilo et al 2010). This would enhance its potential application in medical imaging and material property identification.

\subsection{A basis for vibro-acoustic wave carrying information on the ROI}

The primary effect of ultrasound in a body is to generate a sequence of compression and rarefaction in it, leading to the generation of acoustic waves. An attendant transverse shear wave can arise only as a secondary effect, owing to a non-zero Poisson's ratio and/or material anisotropy. Depending on the magnitude of the mechanical force applied by the ultrasound transducers, the relative amplitude of the shear-induced displacement vis-à-vis its vibro-acoustic counterpart can vary. For the small ultrasound force applied in the present case to prevent overheating 
of the ROI, the amplitudes are small and typically of the same order. In addition, the shear wave, other than being noisy, is also greatly attenuated by the object so that its detection at the object boundary is unlikely. For much larger ultrasound forcing, as in with ablation equipment discussed by Wu et al (2000), shear waves may propagate through the body and hence can be used to image the shear modulus distribution. We emphasize that this propagating shear wave is large enough for detection only because of the high intensity ultrasound used in tissue ablation. This is not so in the vibro-acoustography equipment we have in mind.

In our measurement, as shown below, it is the acoustic wave that acts as the carrier of the spatial signatures of shear-induced dynamics pertaining only to the ROI: the focal volume plus a small neighbourhood of it. To begin with, consider the linear momentum balance equation for the ROI:

$$
\rho \frac{D \dot{\mathbf{u}}}{D t}=-\nabla p+\nabla \cdot \boldsymbol{\sigma}^{\mathrm{dev}}, \mathbf{x} \in \Omega_{\mathrm{ROI}}
$$

where $\dot{\mathbf{u}}$ is the velocity of a material point $\mathbf{x}, \boldsymbol{\sigma}^{\mathrm{dev}}$ is the deviatoric part of the Cauchy stress tensor, $p=p_{0}+\widetilde{p}_{a}$ is the pressure composed of an initial component $p_{0}$ and an ultrasoundgenerated acoustic part $\widetilde{p}_{a} . \Omega \supset \Omega_{\mathrm{ROI}}$ denotes the domain corresponding to the ROI. In what follows, we neglect the effect of $p_{0}$ so that the pressure gradient term on the rhs of equation (1) is given by $\nabla \widetilde{p}_{a}$. Note that $\widetilde{p}_{a}$ picks up the time-modulations of the shear-dominated component of displacement through equation (1) and propagates in the same way (in space and time) through the well-known wave equation:

$$
\frac{1}{c^{2}} \ddot{p}_{a}=\nabla . \nabla p_{a}, \quad \mathbf{x} \in \Omega
$$

Here the acoustic wave velocity is given by $c^{2}=E / \rho$, with $E$ being the Young's modulus and $\rho$ the mass density, which, in turn, satisfies the continuity equation:

$$
\dot{\rho}+\nabla \cdot(\rho \dot{\mathbf{u}})=0 .
$$

In the small deformation setup considered here, the effect of temporal and spatial variations in $\rho$ is neglected, and thus equation (3) does not play a significant role in the arguments that follow. Observing that $\widetilde{p}_{a}$ is expressible as $p_{a} I_{\Omega_{\mathrm{ROI}}}$ (with $I_{\Omega_{\mathrm{ROI}}}$ denoting the indicator function over the ROI), we may split $p_{a}$ as $p_{a}=p_{a}^{0}+p_{a} I_{\Omega_{\mathrm{ROI}}}$ so that equation (2) is recast as:

$$
\frac{1}{c^{2}} \ddot{p}_{a}^{0}-\nabla \cdot \nabla p_{a}^{0}=\nabla \cdot \nabla \widetilde{p}_{a}-\frac{1}{c^{2}} \ddot{\tilde{p}_{a}}, \quad \mathbf{x} \in \Omega \backslash \Omega_{\mathrm{ROI}} .
$$

In view of the fact that ultrasound-induced mechanical vibration in the ROI is governed mainly by the linear momentum balance equation, equation (1), within the ROI, and assuming that the coupling effect of the acoustic wave propagation equation, equation (2), within this region has a negligible effect on the displacement $\mathbf{u}$, the domain of acoustic wave propagation in equation (4) has been restricted accordingly to $\Omega \backslash \Omega_{\mathrm{ROI}}$. Denoting the source term on the rhs of equation (4) by $S(t)$, one readily observes that the shear-induced temporal modulations in the deformation are contained within $S(t)$, which are, in turn, propagated by $p_{a}^{0}$ as acoustic waves outside the ROI. In other words, while we merely detect $p_{a}^{0}(\mathbf{x}, t), \mathbf{x} \in \partial \Omega$ on the object boundary, the Fourier spectrum of the same contains the shear deformation signatures as well as the shape of the ROI because of $I_{\Omega_{\mathrm{RO}}}$ in the source term of equation (4) and the density of the material, as this affects the amplitude of $\widetilde{p}_{a}$, also appearing in the source term. To the best of our understanding, this argument explaining the passage of shear-modulus-induced information 
through an acoustic wave modulation has not been explicated in the vibro-acoustography literature.

Since the VA signal is also affected by a number of other parameters than the elastic properties of the object, a quantitative connection of the signal amplitude to the elastic properties can be established only after carefully accounting for these other contributions to the measured signal. In the inverse problem formulation, the linear momentum balance equation for the ROI is set up and solved for the displacement field, which is used to compute and propagate, through the surrounding fluid, the acoustic pressure it generates. What is not accounted for here, as pointed out earlier, is the variation in the radiation force as the ROI scans the object section to be imaged. This unaccounted variation renders the computed acoustic pressure inaccurate, also forcing the parameter recovery to become erroneous. In this work, we demonstrate an alternative measurement scheme which allows a quick and accurate recovery of the elastic modulus in the ROI: we replace the acoustic amplitude data with one involving frequency - the frequency at which the acoustic amplitude reaching the object surface peaks. We claim, and prove in this work, that this resonant behaviour in measurement corresponds to the resonance of the VROI; i.e. the frequency at which the measured acoustic amplitude peaks correspond to one of the normal modes of the ROI. Because of this, and the fact that the ROI, though surrounded by the rest of the object, may be considered as a freely vibrating body, verification of the measured resonant frequency is achieved with computational expediency by arriving at the normal modes of vibration of the ROI. This measurement is reminiscent of the natural frequency measurement one employs in resonant ultrasound spectroscopy (RUS) from which the elastic constants of objects of interest are recovered (Zadler et al 2004). Since the frequency measurement is almost unaffected by noise in the amplitude owing to the presence of other sources of vibration in the object (Fan and Qiao 2011), the problem of noise in measurements affecting the parameter recovery are nearly eliminated. We have employed ANSYS $^{\circledR}$, a commercial software, to compute the resonant modes of the ROI. In the sub-section below, we briefly explain the variational method employed in the commercial programme to arrive at these frequencies.

\subsection{Computation of natural frequencies of vibration of the focal volume}

Inversion of the resonance modes of vibrating objects for the size, shape, density and elastic properties of the object has been proposed and implemented in a number of publications in the past (Visscher et al 1991, Migliori et al 1993, Zadler et al 2004). Based on the above papers, we give a brief description of how natural frequencies are computed as well as how the measured frequencies can be used to recover the elastic parameters by solving a simple inverse problem. The principle used is the well-known variational approximation for undamped, unforced mechanical systems, leading to an eigenvalue problem to determine the natural frequencies; viz., for a free body, i.e. with a stress-free boundary condition, with the displacement vector, $\mathbf{u}$, satisfying the elastic wave equation, the Lagrangian $L=\int_{V}\left(E_{\mathrm{K}}-E_{\mathrm{P}}\right) \mathrm{d} V$ of the system is stationary. Here $E_{\mathrm{K}}$ is kinetic energy equal to $\frac{1}{2} \rho \omega^{2} u_{i} u_{i}$ and $E_{\mathrm{p}}$ the potential energy equal to $\frac{1}{2} C_{i j k l} \partial_{j} u_{i} \partial_{l} u_{k}$, where the Einstein convention of repeated indices is followed. Moreover, $u_{i}$ etc are components of $\mathbf{u}$ which have harmonic time variations given by $\mathrm{e}^{j \omega t}, \rho$ and $C_{i j k l}$ are the mass density and elasticity tensor respectively. The next step is to expand $u_{i}$ in terms of a basis function set defined over $V, u_{i}=\widetilde{u}_{i, \lambda} B_{\lambda}, \lambda=1,2 \ldots . N$. The principle $\delta L=0$ leads to the following computable equation, which is our symmetric eigenvalue problem:

$$
\omega^{2} \mathbf{M} \cdot \boldsymbol{\alpha}=\boldsymbol{\Gamma} \cdot \boldsymbol{\alpha},
$$


where the mass matrix $\mathbf{M}$ is real, symmetric and positive definite and the stiffness matrix $\Gamma$ is real, symmetric and positive semi-definite. The elements of $\mathbf{M}$ and $\boldsymbol{\Gamma}$ are $M_{i \lambda, i^{\prime} \lambda^{\prime}}=\delta_{i, i^{\prime}} \int_{V} B_{\lambda} \rho B_{\lambda^{\prime}} \mathrm{d} V$ and $\Gamma_{i \lambda, i^{\prime} \lambda^{\prime}}=C_{i j j^{\prime} j^{\prime}} \int_{V} B_{\lambda, j} B_{\lambda^{\prime}, j^{\prime}} \mathrm{d} V$, where $i, i^{\prime}, j, j^{\prime} \in[1,3], \lambda^{\prime}=1,2, \ldots N$ and $B_{\lambda, j}$ denotes the derivative of $B_{\lambda}$ with respect to the $j$ th spatial coordinate. The concatenated (i.e. $3 N$ dimensional) eigenvector $\boldsymbol{\alpha}$ is obtained by arranging all the components $\widetilde{u}_{i, \lambda}$ of displacement $u_{i}$.

The major contribution of Visscher et al (1991) in the development of an easily computable forward model which is applicable for an arbitrarily shaped (and sized) object is the introduction of a basis $B_{\lambda}$ using monomials of the form $B_{\lambda}=x^{l(\lambda)} y^{m(\lambda)} z^{m(\lambda)}$. The $\left\{B_{\lambda}\right\}$ are not an orthonormal set and therefore the matrix $\mathbf{M}$ is not diagonal, an aspect that is not computationally expeditious in the inversion of equation (5). However, since the orthonormal basis has to be chosen afresh for each problem, depending on the shape of the object and the variation of $\rho(\mathbf{r})$, we lose the advantage of a generalized procedure for computing the matrix elements in closed form, valid for all arbitrary shapes. The basis with monomials allows us such closed form, analytic expressions valid for any object, without destroying the symmetry and positive definiteness of $\mathbf{M}$.

The standard package ANSYS employs a variational formulation as above and an expansion in terms of monomials for an eigenvalue analysis of the ROI. For the forward computation of resonant frequencies, we assume that the vibrating ROI has a free boundary; see also the next sub-section. The inputs to ANSYS are (i) the geometry of the vibrating region, delimited by the free boundary, (ii) the material parameters such as $\rho$ and $C_{i j k l}$ (in the present case only Young's modulus and Poisson's ratio, as the elastic region is considered to be linear isotropic (Young's moduli for different phantoms were obtained from the procedure to be discussed later; see section 2(c)), and (iii) the boundary conditions applicable for the ROI.

To identify the free boundary (i.e. where the displacement is zero) we first transport the ultrasound pressure from the surface of the transducer to the focal region, convert the pressure to a force distribution and use it in a momentum balance equation for the entire object. The solution of the momentum balance equation helps us identify the nodes where the displacement is larger than a preset lower bound, and thus delineate the boundary $\partial \Omega$ separating the vibrating region from its non-vibrating complement. The details are in the following section.

\subsection{Numerical study of the dependence of the resonant mode in an agarose slab on its shear modulus}

In order to numerically compute the resonant frequencies of the VROI we need to know $\partial \Omega$. To compute $\partial \Omega$, we solve the linear momentum balance equation for the entire object slab of dimension $20 \mathrm{~mm} \times 30 \mathrm{~mm} \times 40 \mathrm{~mm}$, driven by a sinusoidal forcing at the intersection volume of the focal regions of the two ultrasound transducers. The relatively low-frequency sinusoidal forcing in the ROI is obtained by 'mixing' two ultrasound beams oscillating at frequencies $f_{0}+\Delta f_{0} / 2$ and $f_{0}-\Delta f_{0} / 2$ with the offset $\Delta f_{0}$ adjustable from tens of $\mathrm{Hz}$ to a few $\mathrm{kHz}$. As discussed by Chandran et al (2011), the ultrasound amplitudes from the transducer surface are numerically propagated, solving the Westervelt equation (Kamakura et al 2000), to their focal regions. The inputs for solving the equation are specific to the transducers (http: //sonicconcepts.com) and the phantom used during the actual experiment: the radius of the active surface $=32 \mathrm{~mm}$, the focal length $=62.6 \mathrm{~mm}$, the velocity of sound in the object $=1500 \mathrm{~m} \mathrm{~s}^{-1}$, the average object mass density $=1000 \mathrm{~kg} \mathrm{~m}^{-3}$ and the non-linearity co-efficient, which enters the Westervelt equation, is taken as 3.5, which is close to that reported for water. The computed pressure distribution due to one of the ultrasound transducers is shown in figure 1 . 


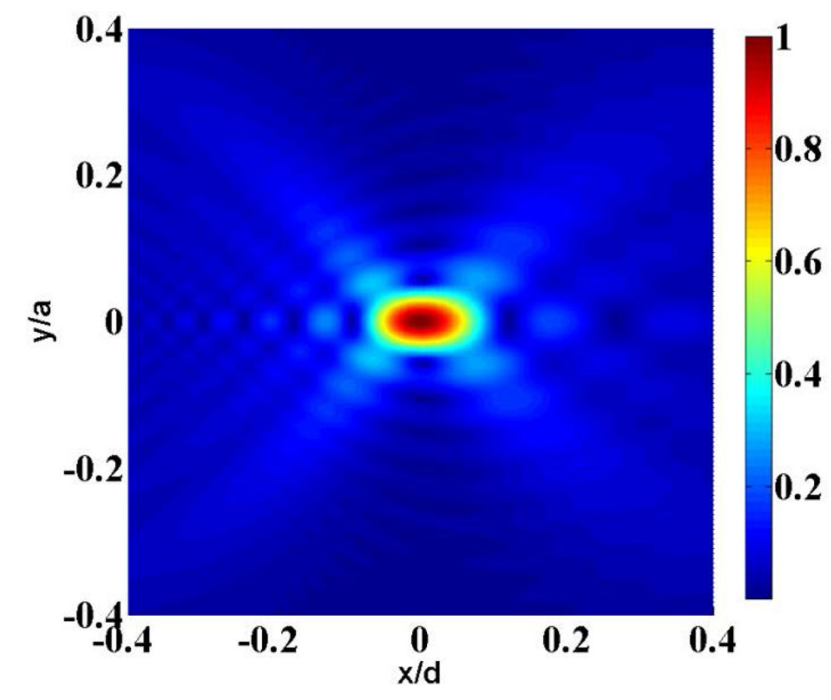

Figure 1. A cross-sectional plot of the normalized pressure distribution in the focal region of the transducer. The cross-section contains the transducer axis $\mathbf{x}$. Here, $\mathbf{a}$ and $\mathbf{d}$ are respectively the diameter and focal length of the transducer with the focus situated at the origin.

The time-averaged intensity of the mixed amplitude is computed first and then the radiation force is obtained, this last step also requiring knowledge of the sound velocity and absorption coefficient of the material of the body and the integration time constant of the detector used. The sinusoidal force in the common focal region is of the form $F(t)=F_{0} \cos \left(2 \pi \Delta f_{0} t\right)$ and is in the direction bisecting the intersecting axes of the transducers. This sinusoidal force distribution, at the beat frequency, is non-zero only over a small region comprising the intersection of the focal volumes of the ultrasound transducers. With this forcing we set up the linear momentum balance equation, which is

$$
\rho \ddot{\mathbf{u}}=\nabla \cdot \sigma+F_{0}(\mathbf{x}) \cos \left(2 \pi \Delta f_{0} t\right) I_{\Omega_{\mathrm{RO} I}} .
$$

Here, $\sigma$ is the Cauchy stress tensor and $\mathbf{x} \in I_{\Omega_{\mathrm{Ror}}}$. We have employed ANSYS to solve equation (6) for one full cycle of the sinusoidal forcing $F(t)$ and obtained a cycle of displacements of the particles in the object, from which the amplitude of vibration is ascertained. The stressfree boundary of the ROI, $\partial \Omega$ is obtained by identifying the boundary nodes that separate the vibrating region from the region surrounding it, which, for all practical purposes, is deemed to be non-vibrating.

For agarose, which is the material used in the objects in the experiments reported here, we have set up equation (6) after computing the ultrasound radiation force in the intersection region of the foci of the ultrasound transducers. The transducers are assumed to have an $f / n o$ of 0.9781 with a designed centre frequency of $1.1 \mathrm{MHz}$ and are driven by a dual-channel sinusoidal voltage signal, one operating at $1.1 \mathrm{MHz}+\Delta f_{0} / 2$ and the other at $1.1 \mathrm{MHz}-\Delta f_{0} / 2$, with the peak-to-peak voltage of both set at $50 \mathrm{~V}$. The difference frequency, $\Delta f$ as stated earlier, can be precisely set from tens of $\mathrm{Hz}$ to $1 \mathrm{kHz}$. These specifications match the transducers used in the experiments of section 3 , with figure 2 showing schematically the orientation of the transducers used during the experiments. For illustration, figure 3 shows the force field in the region surrounding the focal volume, from one of the transducers used, the corresponding plot for the other being identical to the above, but only rotated $90^{\circ}$. We reiterate that the 


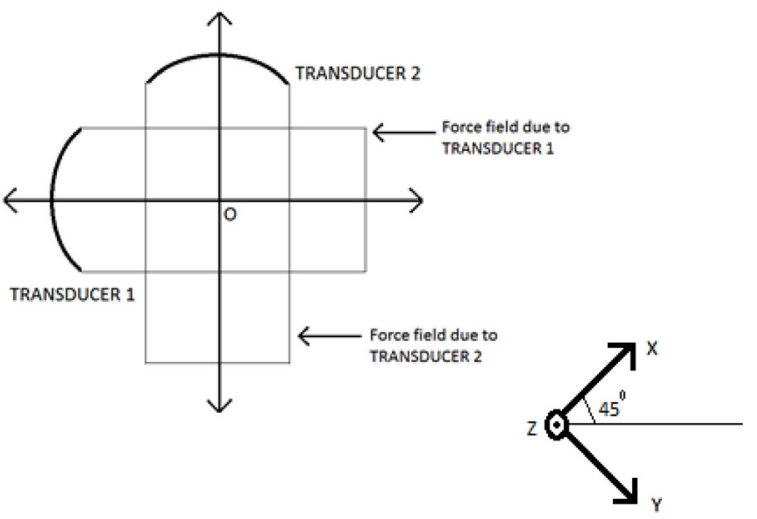

Figure 2. A schematic diagram of the orientation of the two transducers (top view); to compute displacements using the resultant force from the dual transducer system, we used a coordinate system different from those shown in figures 1 and 3, as depicted in the inset.

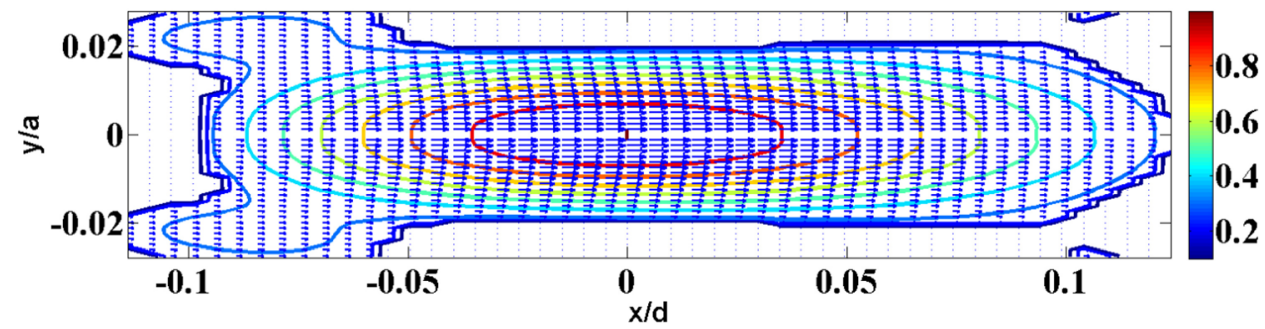

Figure 3. Contour plots of the force distribution along the axial plane for transducer 1 . In this coordinate system, the transducer axis is in the $x$-direction. The contour plots represent decreasing amplitudes, starting from around $-0.4 \mathrm{~dB}$ and ending at $-6 \mathrm{~dB}$ of the maximum. For transducer 2 we get an identical plot, rotated clockwise by $90^{\circ}$.

resultant 'external' force on the body is due to the mixing of intensities in the focal regions and is applied at the non-zero difference frequency maintained between the transducers. Because of this, the external force is non-zero only within the intersection volume of the focal regions of the transducers. With this force assumed to be applied at the centre of an isotropic homogeneous material, mimicking the agarose phantom of the experiment, we arrived at the displacement distribution using an appropriate axis-symmetric analysis using ANSYS. The amplitudes of vibration in three orthogonal planes passing through the centre of the object are shown in figure 4 . We point out that the ensuing dynamics of deformation introduced by the radiation force is indeed local, confined to almost within the region where the force is non-zero. In other words, one may say that the forced vibration response is dominated by eigenvectors that best capture the ROI-localized deformed shape of the object. In order to further demonstrate this point, the eigenvalue problem was solved, as described in the following paragraph, to compute the natural frequencies of the VROI considered to be enclosed by the $-3 \mathrm{~dB},-4.5 \mathrm{~dB},-6 \mathrm{~dB}$ and $-7.5 \mathrm{~dB}$ surfaces of the central vibration amplitude. The computed resonant modes, tabulated in table 1 , are seen to be almost invariant to the extension of the VROI, demonstrating that for all practical purposes, the dynamics is confined to a small region around the ultrasound focal volume. This also justifies imaging the object's mechanical property distribution through the acoustic amplitudes measured when the object 


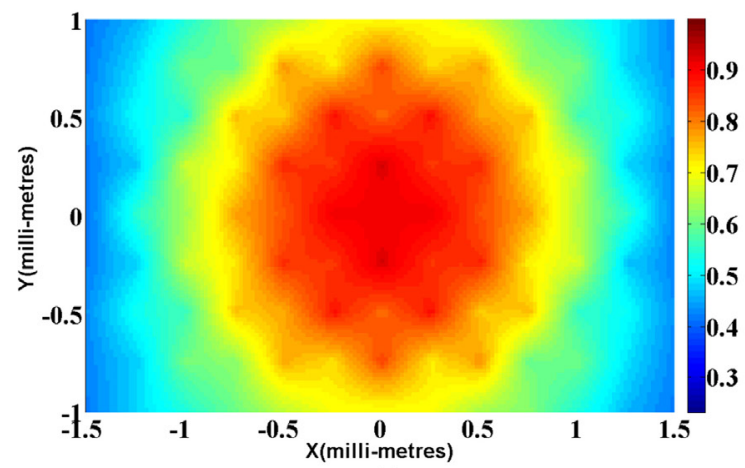

(a)
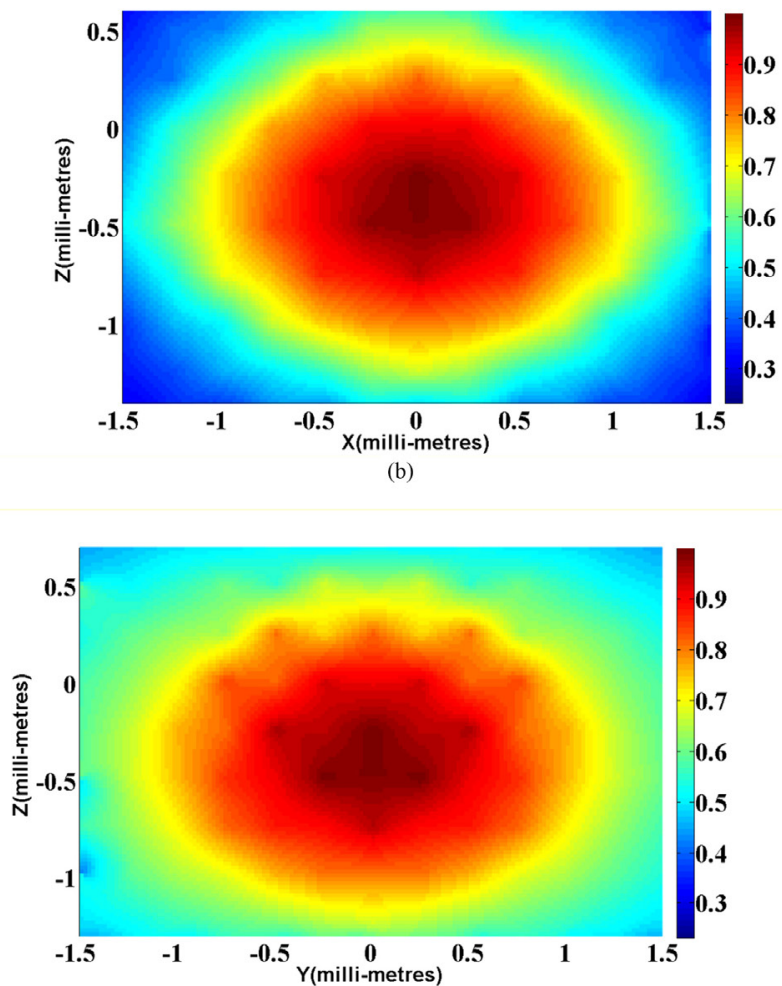

(c)

Figure 4. Axial cross-sections (a) $X-Y$, (b) $X-Z$, (c) $Y-Z$ of the normalized displacement magnitude around the focal volume. It is seen that the displacements go to small values (blue) quickly.

is raster-scanned with the ultrasound focal volume (Alizad et al 2004). Since the stress field and displacement produced by the radiation force are local, confined to the VROI, the acoustic source term driving the pressure wave propagating from the ROI in equation (4) is a function of the local mechanical and acoustic properties of the object.

Having found the geometry of the VROI, we now proceed to compute the natural frequencies of vibration. For this, we set up equation (5), for which the additional inputs are the 
Table 1. A comparison of the experimentally determined first mode of vibration from computer simulations using ANSYS.

\begin{tabular}{|c|c|c|c|c|c|}
\hline $\begin{array}{l}\text { Percentage } \\
\text { of Agarose }\end{array}$ & $\begin{array}{l}\text { Experimentally } \\
\text { measured } \\
\text { first natural } \\
\text { frequency }(\mathrm{Hz})\end{array}$ & $\begin{array}{l}\text { Computed } \\
\text { first natural } \\
\text { frequency }(\mathrm{Hz}) \\
\text { with boundary } \\
\text { from }-3 \mathrm{~dB} \\
\text { points }\end{array}$ & $\begin{array}{l}\text { Computed } \\
\text { first natural } \\
(\mathrm{Hz}) \text {, with } \\
\text { boundary } \\
\text { from }-4.5 \mathrm{~dB} \\
\text { points }\end{array}$ & $\begin{array}{l}\text { Computed } \\
\text { first natural } \\
\text { frequency }(\mathrm{Hz}) \text {, } \\
\text { with boundary } \\
\text { from }-6 \mathrm{~dB} \\
\text { points }\end{array}$ & $\begin{array}{l}\text { Computed } \\
\text { first natural } \\
\text { frequency } \\
(\mathrm{Hz}), \text { with } \\
\text { boundary } \\
\text { from }-7.5 \\
\text { dB points }\end{array}$ \\
\hline 1 & 260 & 243 & 240 & 238 & 237 \\
\hline 2 & 350 & 340 & 336 & 334 & 333 \\
\hline 3 & 410 & 384 & 380 & 377 & 375 \\
\hline 4 & 450 & 446 & 441 & 437 & 435 \\
\hline
\end{tabular}

density and (visco) elastic distribution in the ROI (homogeneous in the present case), and invert it for $\left\{\omega^{2}\right\}$, the eigenvalues of the vibrating region. As stated earlier, this was accomplished through a modal analysis subroutine of ANSYS. When the percentage of agarose (by weight) in the solution used to prepare the phantom is varied from 1 to 4 , the storage modulus, as verified by rheometer measurements in the experimental section (section 3), varied from approximately $20-60 \mathrm{kPa}$. The rheometer measurements also give us the loss modulus, $G^{\prime \prime}$. Within the assumption of small strain, a model that may perhaps be considered to reasonably describe the behaviour of human tissue upon loading, is the Kelvin-Voigt model (Dooubal et al 2004, Wu 2005), under which it can easily be shown that the measured storage modulus is numerically equal to the shear modulus $(G)$ of the material (Barnes et al 1989). Young's modulus is three times the shear modulus for the present value of Poisson's ratio (0.495). Figures 5(a) and (b) respectively, give the variation in $G^{\prime}$ and $G^{\prime \prime}$ plotted against the amplitude of strain applied by a rheometer for different samples with a varying percentage of agarose. The corresponding variation of the computed first fundamental frequency of the VROI with $G$ is given in figure 6. It is seen that for the storage modulus range we are interested in, the variation of the corresponding fundamental frequency with $G^{\prime}$ is almost linear. When we try to fit a second degree polynomial, $\omega=a_{0}+a_{1} G^{\prime}+a_{2} G^{\prime 2}$ to represent this variation, the coefficients $a_{0}, a_{1}$ and $a_{2}$ are estimated to be $130.356,7.547$ and -0.034 respectively. The variation shown in figure 6 is also verified by the experimentally measured peak in the detected acoustic amplitude, as $\Delta f_{0}$ is swept along a range of frequencies in which we guessed where the natural frequency of the ROI should lie from their computed values shown in figure 6.

Having established a connection between the resonant modes of the VROI, available in the VA wave originating therein and detected outside the object, and the local $G$ of the material in the VROI, the question of inversion of the measured resonant modes for $G$ becomes pertinent. We present the results of the material property inversion from both the simulated and experimental natural frequency data. In passing, note that for resonant mode calculation other parameters of the VROI are required, such as the density of the material and its shape and volume, apart from $G$. The shape and volume can only be approximated by assuming $\mathrm{a}-3 \mathrm{~dB}$ surface of maximum displacement separating the vibrating region from the rest of the body. This introduces an error, perhaps small, in our 'forward' calculation, and consequently in the inversion as well. We have not attempted to quantify said error in this work. The second minor issue is that in an inhomogeneous medium, the recovered $G$ is a spatial average for the VROI. 


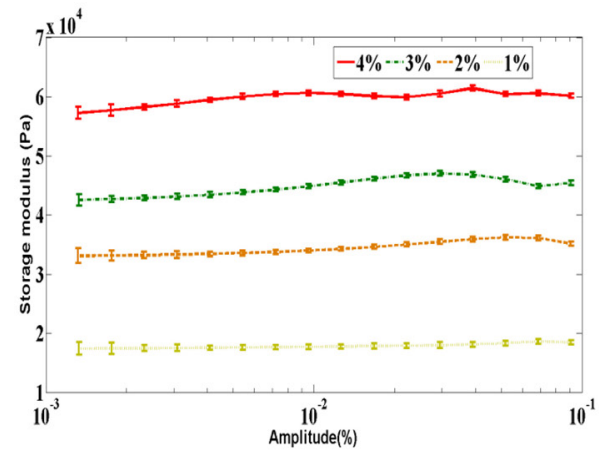

(a)

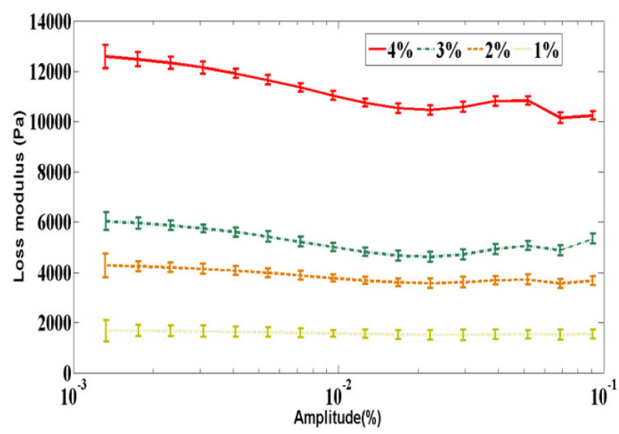

(b)

Figure 5. The experimentally obtained variations of the storage modulus $\left(G^{\prime}\right)$ (a), and loss modulus $\left(G^{\prime \prime}\right)$ (b), with strain from the Anton Paar rheometer for samples with $1 \%$, $2 \%, 3 \%$, and $4 \%$ (by weight) of agarose powder.

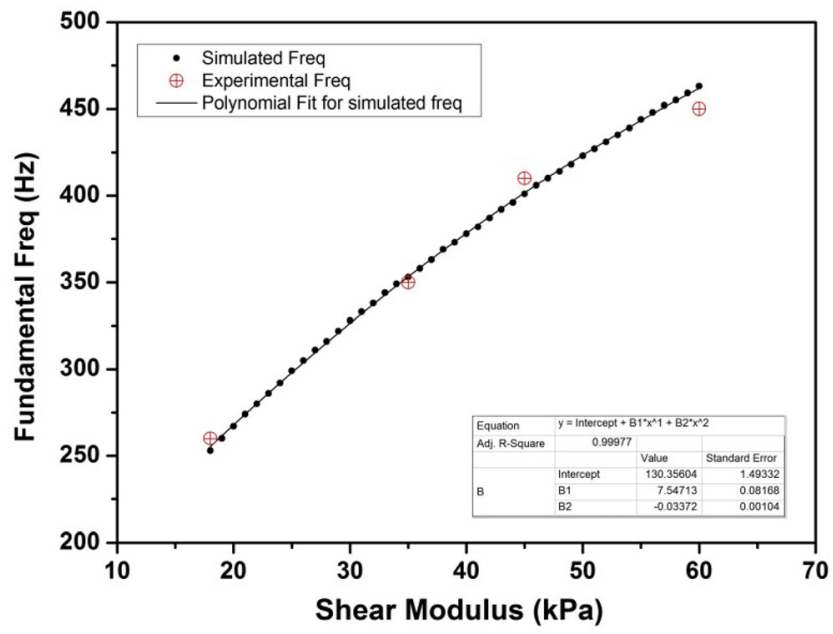

Figure 6. Variation of the first modal frequency, both simulated and experimental, with the shear modulus of the material in the ROI.

\section{Experimental determination of the first natural frequency of the vibrating region in an agarose phantom}

\subsection{Preparation of the phantom}

Agarose powder (Sigma Aldrich A9539) is added to distilled water kept at $40{ }^{\circ} \mathrm{C}$, and the mixture is constantly stirred until the powder is completely dissolved. The solution is slowly cooled to room temperature in moulds of the required shape to solidify to the shape of the container. We prepared slabs of dimension $2 \mathrm{~cm} \times 3 \mathrm{~cm} \times 4 \mathrm{~cm}$ with the weight percentage of agarose powder increasing from 1 to 4 in steps of one. As the percentage of agarose powder increased, so did the storage modulus of the resulting slab; the variation in the measured $G^{\prime}$ and $G^{\prime \prime}$ with the percentage of agarose powder is shown in figures 5(a) and (b) respectively. As observed earlier, the $G^{\prime}$ obtained is numerically equal to the shear modulus. 


\subsection{Experimental setup for determining the natural frequencies}

The experimental setup is shown schematically in figure 7, and has three parts: (1) a pair of ultrasound transducers to provide low-frequency sinusoidal forcing at its intersecting focal volume; (2) the object mounted on translation stages immersed in acoustic impedance matching liquid and (3) the acoustic amplitude detector, which in our case is the FBG mounted suitably on a cantilever.

The ultrasound transducers (Sonic Concepts, Washington) operate at a centre frequency of $1.1 \mathrm{MHz}$, a combination of which delivers an average power of $15 \mathrm{~mW}$ concentrated at the focal region that accounts for a temporally averaged intensity of $5.736 \mathrm{~mW} \mathrm{~mm}^{-2}$. They are driven by a dual-channel function generator (Tektronix, AFG, 3022B) after suitable power amplification. The operating frequency of one of the transducers can be altered precisely to $1.1 \mathrm{MHz}+\Delta f_{0} / 2$ and the other to $1.1 \mathrm{MHz}-\Delta f_{0} / 2$, where $\Delta f_{0}$ can be varied from tens of $\mathrm{Hz}$ to $1 \mathrm{kHz}$. The transducers are of the identically focusing variety with $\mathrm{f} / \mathrm{no}$. of 0.9781 , precisely aligned so that their focal volumes intersect at an angle of $90^{\circ}$ at their thinnest waist region. The volume of the intersecting region is found to be approximately $4.23 \mathrm{~mm}^{3}$, which imposes the spatial resolution limit on the mechanical property images we may recover.

The object is the agarose slab prepared earlier: four samples with shear moduli of 17949.4 $\mathrm{Pa}, 35082.7 \mathrm{~Pa}, 45281.1 \mathrm{~Pa}$ and 59983.9 $\mathrm{Pa}$. These values are selected keeping in mind the shear modulus of cancerous lesions in human breast tissue. For a typical experiment, one of the slabs is mounted on a computer-controlled translation stage (Holmarc, Cochin, India), with three translational and two rotational degrees of freedom, and aligned so that the intersecting volume of the ultrasound focal volumes 'interrogate' the region in the slab we want to image. The object is immersed in a water tank for acoustic impedance matching. To produce a cross-sectional, shear modulus image of, say, an excised organ, the required cross-section is raster-scanned with the intersecting region of the ultrasound force in the focal regions.

The low-frequency acoustic beam, emanating from the ROI and reaching the object surface, is detected by an FBG sensor for pressure. The sensing element, an FBG mounted on a cantilever towards its fixed end with the cantilever tip (the free end) touching the surface of the agarose slab, is also immersed in the water bath (figure 7). The procedure adopted for in-house fabrication of the FBG sensor has been described by Hill et al (1993), Othonos and Kalli (1999) and Kashyap (1999). The cantilever is a strip of stainless steel with dimensions $5 \mathrm{~mm} \times 20 \mathrm{~mm} \times 0.1 \mathrm{~mm}$. When the acoustic pressure reaches the surface of the object and pushes the free end of the cantilever, a stress field is developed on its surface. The FBG responds to the strain developed, producing a proportional shift in its support wavelength. (For further details on how an FBG sensor works, see appendix.) The electronic assembly, which forms part of the FBG interrogator (Micron Optics sm130-700), detects this shift in wavelength to the $\mathrm{pm}$ resolution, which is the readout from the sensor. For the relatively lowintensity pressures we are dealing with in the present experiments, the shift in wavelength is considered proportional to the amplitude of the acoustic pressure that the detector receives. The temporal variation of the received acoustic pressure produces a temporal variation in the support wavelength readout by the interrogator (figure 8(a)). The Fourier transform of the time varying signal from the interrogator contains-apart from the peaks at frequencies corresponding to spurious signals picked up and noise - a peak corresponding to the dominant sinusoidal signal in the acoustic wave at frequency $\Delta f_{0}$. The amplitude of this peak is the measurement we make in the present experiments (figure 8(b)).

We have repeated the experiment by fixing the detector at different locations around the object, for example along a line intersecting the transducer axes and then perpendicular to it. There were small changes noticed in the amplitude of the detected signal, but they were small 


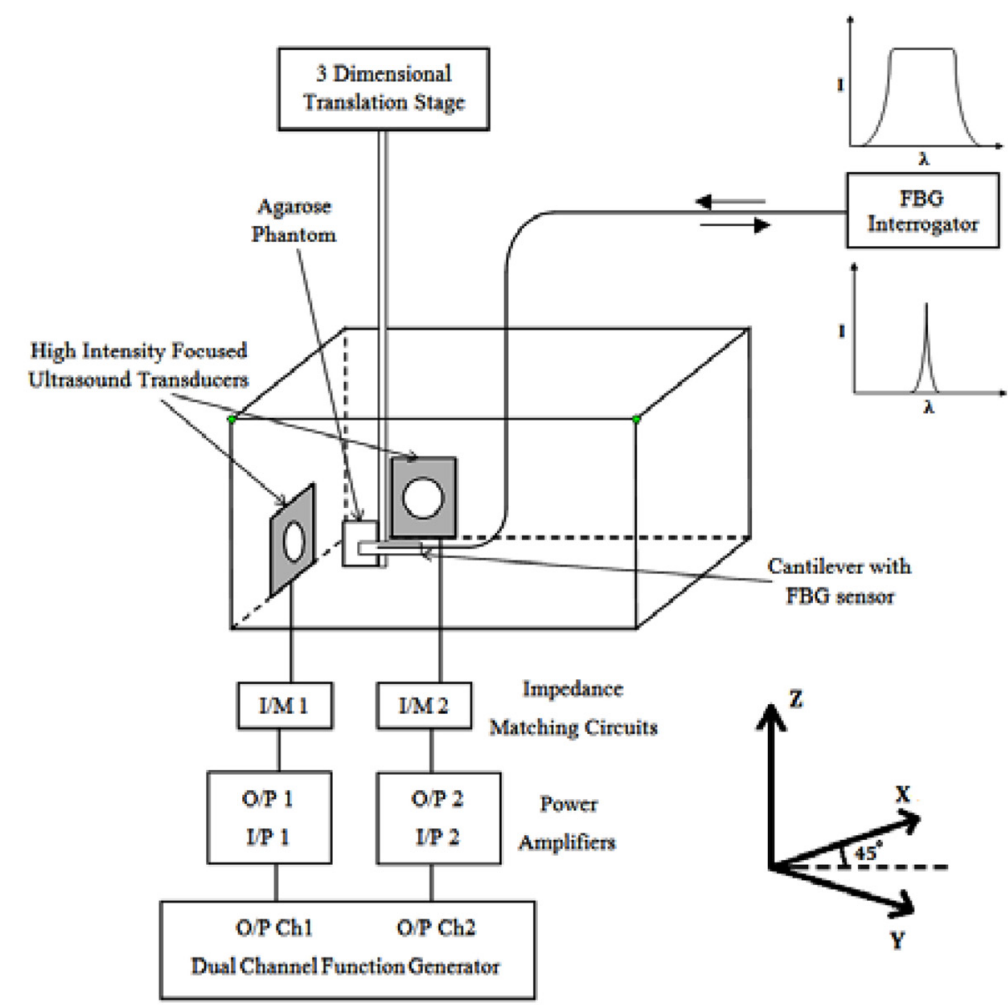

Figure 7. A schematic diagram of the experimental set-up. The two transducers are driven by signals from an ultra-stable, dual-channel function generator after power amplification. The transducers and object are mounted on translation stages. The object and transducers are immersed in a water bath for acoustic impedance matching. The acoustic wave is detected by an FBG sensor mounted on a cantilever.

enough to be ignored. However, the power spectrum revealed that the location of the spectral peaks had not shifted. The amplitude finally used for plotting figure 9 was the mean from such repeated measurements.

\subsection{Experimental determination of the first natural frequency}

In the present experiments the agarose slabs are homogenous. Our objective is to find the resonant mode(s) of the VROI by measuring the frequency at which the measured acoustic amplitude reaching the object surface peaks. For this, the magnitude of the Fourier transform at the ultrasound forcing frequency, $\Delta f_{0}$ of the detected FBG signal is measured as the frequency is swept. Plots of the Fourier transform magnitude versus $\Delta f_{0}$ are shown in figure 9 for slabs with a percentage of agarose varying from 1 to 4 . The peaks observed in figure 9 are our data, which are, in this case, the first natural frequencies of the vibrating ROI. Had we scanned over a larger range of $\Delta f_{0}$, we would have observed other peaks corresponding to higher modes. Unfortunately, the FBG interrogator (Micron Optics sm130-700) used has a high frequency cut-off at $500 \mathrm{~Hz}$, and therefore, we could only measure the first natural frequency. Table 1 gives the experimentally measured frequencies as well as the theoretically computed first natural frequencies for the four slabs with boundaries delineated by the $-3 \mathrm{~dB},-4.5 \mathrm{~dB}$, 


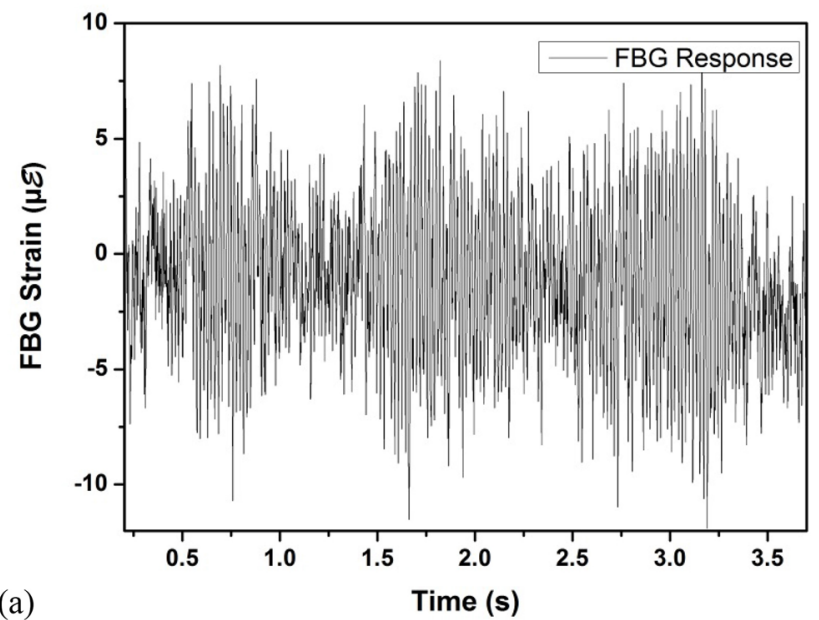

(a)

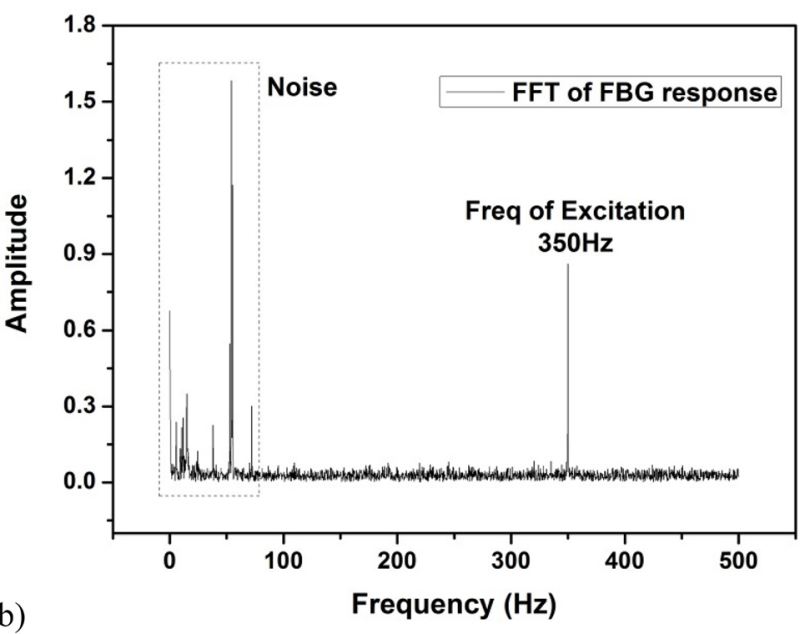

Figure 8. (a) The strain as read out by the sensor owing to the acoustic wave incident on the cantilever tip. (b) The power spectral density of the data of figure (a). The amplitude at the difference frequency, here $350 \mathrm{~Hz}$, is the measurement.

$-6 \mathrm{~dB}$ and $-7.5 \mathrm{~dB}$ displacement decay contours. It is seen that the match between the two is quite good, except perhaps for one value of the shear modulus.

\section{Recovery of shear modulus and discussion of results}

Since we have a reliable route to get the computed counterpart of the experimental measurement, we should be able to recover an unknown modulus of the ROI from the measured natural frequency. Biological objects, such as human organs, with pathological changes brought about by malignancy, are better modelled as orthotropic with the elastic material constitution characterized by a nine-component tensor. Knowledge of these constituent parameters and their variation with the passage of time can throw light on the progression of cancer and the efficacy of treatment modalities. However, recovery of a larger set of parameters requires a larger set of measurements; for example, not just one, but as many resonant modes of the ROI as possible. 


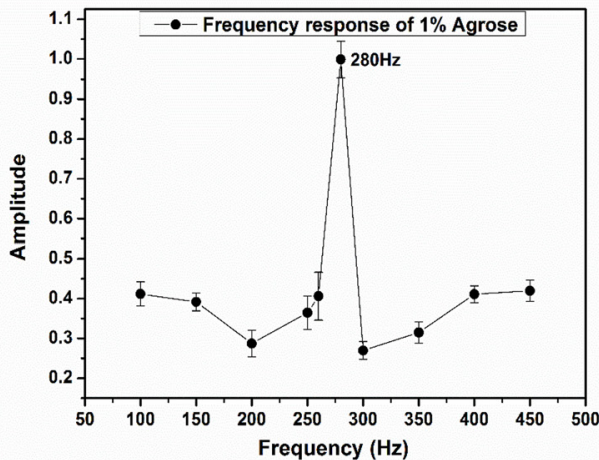

(a)

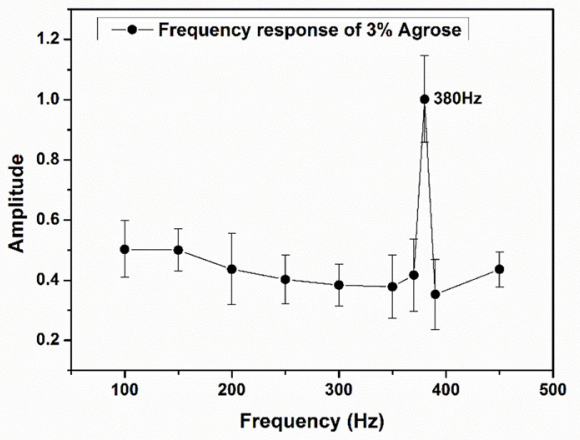

(c)

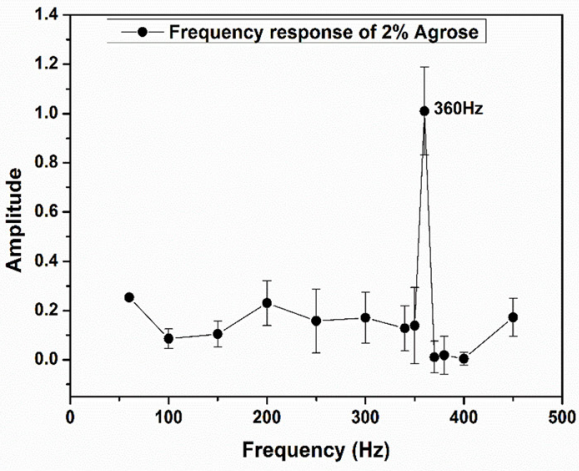

(b)

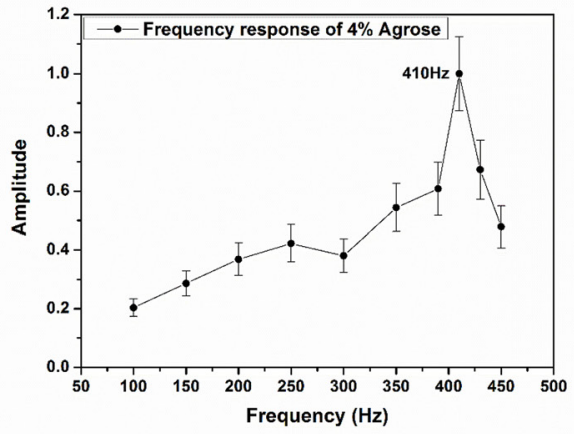

(d)

Figure 9. Variation of the measured power-spectral amplitude at $\Delta f_{0} \mathrm{~Hz}$ (obtained from figure 8(a)) with $\Delta f_{0}$. The peak and its variation with object stiffness, as indicated by the percentage of agarose powder is evident; (a) 1\% agarose, (b) $2 \%$ agarose, (c) $3 \%$ agarose and (d) $4 \%$ agarose.

With a set of natural frequencies as a measurement, and with equation (5) as a forward model to arrive at these frequencies, given the elastic tensor and other mechanical and shape parameters, one can set up a non-linear optimization problem to minimize the mean-square error between the measured and computed natural frequencies. Solving this optimization problem using an appropriate scheme (Teresa et al 2014, Sarkar et al 2015) should recover the elasticity components. However, with our experimental data of only the first natural frequency, we aim to recover a single parameter - the shear modulus corresponding to an isotropic object, which is the agarose phantom. For this single parameter recovery, we use a simple procedure: the method of bisection (Chandran et al 2011).

With the experimentally measured resonant modes, $\Delta f_{m}\left(\Delta f_{m}=260 \mathrm{~Hz}, 350 \mathrm{~Hz}, 410 \mathrm{~Hz}\right.$ and $450 \mathrm{~Hz}$ for the four slabs) we guess two values of the shear modulus, $G_{1}$ and $G_{2}$ such that $G_{1}<G_{m}<G_{2}$, where $G_{m}$ is the unknown modulus to be found. Using the forward equation, resonant frequencies $\Delta f_{1}$ and $\Delta f_{2}$ corresponding to $G_{1}$ and $G_{2}$, are computed. After ascertaining whether $\Delta f_{m}$ is in the interval between $\Delta f_{a}=\frac{\Delta f_{1}+\Delta f_{2}}{2}$ and $\Delta f_{i}, i=j ; j=1,2$, the search interval for $G$ is reduced to lie between $G_{a}=\left(G_{1}+G_{2}\right) / 2$ and $G_{j}$. This procedure is continued until the computed resonant frequencies match the measured ones within the tolerance specified. For the four slabs, the recovered $G$ values are given in table 2 along with the values obtained from the rheometer. It is observed that the match between the two is quite good. 
Table 2. Verification of the shear modulus values obtained by the present method through standard rheometer measurements.

\begin{tabular}{lll}
\hline $\begin{array}{l}\text { Percentage of } \\
\text { agarose }(\%)\end{array}$ & $\begin{array}{l}\text { Experimentally measured values of } \\
\text { shear modulus from rheometer }(\mathrm{Pa})\end{array}$ & $\begin{array}{l}\text { Reconstructed values of } \\
\text { shear modulus }(\mathrm{G})(\mathrm{Pa})\end{array}$ \\
\hline 1 & $17949( \pm 1093)$ & 18748.4 \\
2 & $35082( \pm 1514)$ & 34385.8 \\
3 & $45281( \pm 985)$ & 46866.7 \\
4 & $59983( \pm 1125)$ & 56734.3 \\
\hline
\end{tabular}

The good match between the computed first natural frequency of vibration of the ROI and the experimentally measured peak in the acoustic spectrum emanating from the vibrating region, and the consequent match in the recovered shear modulus with that measured by the rheometer, has ensured an easily implementable quantitative vibro-acoustography technique to characterize tissue on the basis of its elastic modulus. Since it is a frequency-based method, the uncertainties associated with an amplitude measurement, owing to noise, are eliminated. We have not yet proven the efficacy of this procedure in the case of an inhomogeneous visco-elastic distribution, for example in the case of a dissected organ with tumour. The malignancy, as indicated earlier, is characterized not by an isotropic elasticity, but more accurately by an orthotropic assumption, which means the ROI is characterized by a ninecomponent elasticity tensor. The present method, with the assumption that the dissipation of the acoustic wave is not high, can measure many natural frequencies associated with the ROI. From the measured set of natural frequencies, assisted by the additional measurement of mode shapes (Migliori et al 1993, Zadler et al 2004), it should be possible to recover all nine components.

The primary high-frequency acoustic intensity reaching the focal region of the transducers depends on the acoustic absorption and scattering of the intervening medium-the object background. This would make the magnitude of the radiation force applied dependent on the location of the object scanned by the intersecting focal regions. Consequently the acoustic amplitude will be a function of the spatial location of the 'pixel' in the object imaged, and the fidelity of the acoustic amplitude to represent the mechanical property of the object becomes questionable. Here, the frequency-based measurement has an edge relating to the fact that the normal modes (or the locations of the peaks) are not affected by the magnitude of the radiation force applied at the location. The measured fundamental frequency is easily inverted to a quantitative image representing the elastic modulus of the ROI. In table 1, a slight discrepancy is noticed between the computed and experimentally measured resonant modes. Since the computation of the frequency is also dependent on the volume and shape of the vibrating ROI, an error in the evaluation of the size and shape of the ROI affects the numerical accuracy of the computed resonant frequency.

The acoustic spectrum as detected by the FBG sensor is the spectrum of the VROI multiplied by the 'transfer function' of the intervening medium, in our case the background object, denoted as $H(\omega)$ by Fatemi and Greenleaf (1999). Fidelity of the natural frequency readout from the measurement is based on the assumption that $H(\omega)$ does not shift the support of the natural frequencies. Since spectral shift is associated with modulation in the time domain, we anticipate a shift in the locations of the modes only when the intervening medium has periodic 'grating-like' structures in them imposing modulation on the acoustic wave. Since it is uncommon to have such modulations imposed by the background tissue, we assume that the frequency readout truly represents the natural frequencies of the ROI 


\section{Conclusions}

We have demonstrated the measurement of the normal vibrational modes of a region of interest within an object marked by the focal volumes of two ultrasound transducers, from its vibro-acoustic spectrum measured conveniently at its surface. Using the momentum balance equation of the insonified ROI which is coupled with the propagation equation for the acoustic wave, the latter is shown to have a source term modulated by the shear-induced displacement in the ROI. This explains how the acoustic wave can carry local shear information to the boundary. Using the eigenvalue problem governing the resonance of the vibrating region, the natural frequencies of the region are determined. From the measurement and the computation of natural frequencies based on the eigenvalue problem, an easily implementable inversion procedure for recovering the shear modulus of the region is demonstrated. Thus, computationally expedient quantitative vibro-acoustography is proposed, which uses resonant ultrasound spectroscopy - proven earlier for material property recovery in a number of applications - tailored for a free vibrating remote region inside the object. The present frequency-based method is unaffected by the transfer function multiplying the acoustic spectrum, which means that the quantification and compensation of this transfer function are unnecessary. Even though it affects the spectral amplitude, variation of the force distribution does not seem to affect the locations of the natural frequencies when the ultrasound focal volume scans the cross-section of the object. For sensing the acoustic wave, an FBG sensor mounted on a cantilever is used.

Since the interrogator associated with the FBG sensor has a frequency cut-off at $500 \mathrm{~Hz}$, in our experiments we were only able to measure the first fundamental mode of the vibrating ROI. With a very small dissipation component, the acoustic wave suffers little attenuation over a large range of frequencies. Making use of a sensor having an appropriately high cut-off frequency, it should be possible to locate and measure a large number of resonant modes. With a large set of these modal frequencies, and possibly the modal shapes, it should be possible to extend the present technique to the reconstruction of an inhomogeneous distribution of elastic property in the ultrasound focal volume scanning an isotropic body-or more ambitiously, assuming an orthotropic medium, the distribution of a nine-component elasticity tensor. The achievement of this final aim will have far-reaching effects on medical diagnostic imaging.

\section{Acknowledgment}

The authors acknowledge financial support from Defense Research and Development Organization (DRDO), Government of India through Grant No. ERIP/ER/1201130/M/01/109/D(R\&D).

\section{Appendix}

\section{A.1. Principle of fibre Bragg grating sensor}

The FBG is a periodic modulation of the refractive index of the core of a single mode photosensitive optical fibre along its axis. FBGs are photo-inscribed (Meltz et al 1989, Hill and Meltz 1997) by exposing the core of a photosensitive, single-mode, germano-silicate fibre to an interference pattern created by a $248 \mathrm{~nm}$ UV laser beam through a phase mask (Hill and Meltz 1997). When broadband light is launched into an FBG sensor, one particular wavelength $\left(\lambda_{\mathrm{B}}\right)$, which satisfies the Bragg condition (A.1), is reflected back and the rest are transmitted through the fibre. The reflected wavelength from the FBG satisfies the Bragg condition:

$$
\lambda_{\mathrm{B}}=2 n_{\mathrm{eff}} \Lambda
$$


We note that the Bragg resonance wavelength $\lambda_{\mathrm{B}}$ is the free-space centre-wavelength of the input light. Here, $n_{\mathrm{eff}}$ is the effective refractive index of the fibre and $\Lambda$ is the spacing between the gratings. In the present work, FBG sensors with a gauge length of $3 \mathrm{~mm}$ are fabricated in photo-sensitive germania-doped silica fibre, using the phase mask grating inscription method (Hill and Meltz 1997). It is seen from (A.1) that the reflected Bragg wavelength depends on the effective refractive index of the fibre $\left(n_{\text {eff }}\right)$ and the periodicity of the grating $(\Lambda)$ (Hill et al 1993, Othonos and Kalli 1999, Kashyap 1999). The reflected wavelength shift of the FBG sensor with strain is given by Hill et al 1993

$$
\Delta \lambda_{\mathrm{B}}=\lambda_{\mathrm{B}}\left[1-\frac{n_{\mathrm{eff}}^{2}}{2}\left[p_{12}-\nu\left(p_{11}+p_{12}\right)\right]\right] \varepsilon_{z}
$$

where $p_{11}$ and $p_{12}$ are components of the strain-optic tensor, $\nu$ is Poisson's ratio and $\varepsilon_{z}$ is the axial strain change (Tahir et al 2006). The strain sensitivity of an FBG inscribed in a germanium-doped silica fibre is approximately $1.20 \mathrm{pm} / \mu \varepsilon$ (Melle et al 1993).

\section{References}

Aguilo M A, Aquino W, Brigham J C and Fatemi M 2010 An inverse problem approach for elasticity imaging through vibroacoustics IEEE Trans. Med. Imaging 29 1012-21

Alizad A, Greenleaf J F and Fatemi M 2007 Selected applications of dynamic radiation force of ultrasound in biomedicine 11th Mediterranean Conf. on Medical and Biomedical Engineering and Computing 2007 (Berlin: Springer) pp 1021-4

Alizad A, Wold L E, Greenleaf J F and Fatemi M 2004 Imaging mass lesions by vibro-acoustography: modeling and experiments IEEE Trans. Med. Imaging 23 1087-93

Barannik E A, Girnyk A, Tovstiak V, Marusenko A I, Emelianov S Y and Sarvazyan A P 2002 Doppler ultrasound detection of shear waves remotely induced in tissue phantoms and tissue in vitro Ultrasonics 40 849-52

Barnes H A, Hutton J F and Walters K 1989 An Introduction to Rheology vol 3 (Amsterdam: Elsevier) www.sonicconcepts.com/images/pdf/h-101_datasheet.pdf

Bernal M, Nenadic I, Urban M W and Greenleaf J F 2011 Material property estimation for tubes and arteries using ultrasound radiation force and analysis of propagating modes J. Acoust. Soc. Am. 129 1344-54

Brigham J C, Aquino W, Mitri F G, Greenleaf J F and Fatemi M 2007 Inverse estimation of viscoelastic material properties for solids immersed in fluids using vibroacoustic techniques J. Appl. Phys. 101023509

Chandran R S, Roy D, Kanhirodan R, Vasu R M and Devi C U 2011 Ultrasound modulated optical tomography: Young's modulus of the insonified region from measurement of natural frequency of vibration Opt. Express 19 22837-50

dos Santos F L M, Peeters B, Gielen L, Desmet W and Góes L C S 2015 The use of fiber Bragg grating sensors for strain modal analysis Topics in Modal Analysis vol 10 (New York: Springer) pp 93-101

Ďoubal S, Klemera P, Semecký V, Lamka J and Kuchařová M 2004 Non-linear mechanical behavior of visco-elastic biological structures, measurements and models Biophys. Days 47 297-300

Fan W and Qiao P 2011 Vibration-based damage identification methods: a review and comparative study Struct. Health Monit. 10 83-111

Fan X, Case E D, Yang Q and Nicholas J D 2013 Room temperature elastic properties of gadolinia-doped ceria as a function of porosity Ceram. Int. $396877-86$

Fatemi M and Greenleaf J F 1998 Ultrasound-stimulated vibro-acoustic spectrography Science 280 82-5

Fatemi M and Greenleaf J F 1999 Vibro-acoustography: an imaging modality based on ultrasoundstimulated acoustic emission Proc. Natl Acad. Sci. 96 6603-8

Golinval G K J C Experimental modal analysis (http://www.ltas-vis.ulg.ac.be/cmsms/uploads/File/ Mvibr_notes.pdf)

Heikkilä J, Curiel L and Hynynen K 2010 Local harmonic motion monitoring of focused ultrasound surgery—a simulation model IEEE Trans. Biomed. Eng. 57 185-93 
Hill K O and Meltz G 1997 Fiber Bragg grating technology fundamentals and overview J. Lightwave Technol. 15 1263-76

Hill K O, Malo B, Bilodeau F, Johnson D C and Albert J 1993 Bragg gratings fabricated in monomode photosensitive optical fiber by UV exposure through a phase mask Appl. Phys. Lett. 62 1035-7

Huber T M, Fatemi M, Kinnick R and Greenleaf J 2006 Noncontact modal analysis of a pipe organ reed using airborne ultrasound stimulated vibrometry J. Acoust. Soc. Am. 119 2476-82

Ji L and McLaughlin J 2003 Recovery of the Lamé parameter $\mu$ in biological tissues. Inverse Problems 201

Kamakura T, Ishiwata T and Matsuda K 2000 Model equation for strongly focused finite-amplitude sound beams J. Acoust. Soc. Am. 107 3035-46

Kashyap R 1999 Fiber Bragg Gratings (New York: Academic)

Kinney J H, Gladden J R, Marshall G E A, Marshall S J, So J H and Maynard J D 2004 Resonant ultrasound spectroscopy measurements of the elastic constants of human dentin J. Biomech. 37 437-41

Konofagou E E and Hynynen K 2003 Localized harmonic motion imaging: theory, simulations and experiments Ultrasound Med. Biol. 29 1405-13

Konofagou E E, Ottensmeyer M, Agabian S, Dawson S L and Hynynen K 2004 Estimating localized oscillatory tissue motion for assessment of the underlying mechanical modulus Ultrasonics 42 951-6

Kranjc T, Slavič J and Boltežar M 2016 A comparison of strain and classic experimental modal analysis J. Vib. Control 22 371-81

Madsen E L, Sathoff H J and Zagzebski J A 1983 Ultrasonic shear wave properties of soft tissues and tissue-like materials J. Acoust. Soc. Am. 74 1346-55

Melle S M, Alavie A T, Karr S, Coroy T, Liu K and Measures R M 1993 A Bragg grating-tuned fiber laser strain sensor system IEEE Photonics Technol. Lett. 5 263-6

Meltz G, Morey W and Glenn W H 1989 Formation of Bragg gratings in optical fibers by a transverse holographic method Opt. Lett. 14 823-5

Migliori A, Sarrao J L, Visscher W M, Bell T M, Lei M, Fisk Z and Leisure R G 1993 Resonant ultrasound spectroscopic techniques for measurement of the elastic moduli of solids Physica B 183 1-24

Mitri F G and Kinnick R R 2012 Vibroacoustography imaging of kidney stones in vitro IEEE Trans. Biomed. Eng. 59 248-54

Mitri F G, Davis B J, Alizad A, Greenleaf J F, Wilson T M, Mynderse L A and Fatemi M 2008 Prostate cryotherapy monitoring using vibroacoustography: preliminary results of an ex vivo study and technical feasibility IEEE Trans. Biomed. Eng. 55 2584-92

Mitri F G, Trompette P and Chapelon J Y 2003 Detection of object resonances by vibro-acoustography and numerical vibrational mode identification J. Acoust. Soc. Am. 114 2648-53

Nightingale K R, Palmeri M L, Nightingale R W and Trahey G E 2001 On the feasibility of remote palpation using acoustic radiation force J. Acoust. Soc. Am. 110 625-34

Othonos A and Kalli K 1999 Fiber Bragg Gratings: Fundamentals and Applications in Telecommunications and Sensing (Boston, MA: Artech House)

Sarkar S, Roy D and Vasu R M 2015 A global optimization paradigm based on change of measures R. Soc. Open Sci. 2150123

Schwarz B J and Richardson M H 1999 Experimental modal analysis Proc. of the CSI Reliability Week (Orlando, FL, USA) (http://www.maintenance.org/fileSendAction/fcType/0/fcOid/39959094 2963653618/filePointer/399590942964787702/fodoid/399590942964787700/28__Experimental_ Modal_Analysis.pdf)

Tahir B A, Ali J and Rahman R A 2006 Fabrication of fiber grating by phase mask and its sensing application J. Optoelectron. Adv. Mater. 81604

Teresa J, Venugopal M, Roy D, Vasu R M and Kanhirodan R 2014 Diffraction tomography from intensity measurements: an evolutionary stochastic search to invert experimental data JOSA A 31 996-1006

Visscher W M, Migliori A, Bell T M and Reinert R A 1991 On the normal modes of free vibration of inhomogeneous and anisotropic elastic objects J. Acoust. Soc. Am. $902154-62$

Wu T, Felmlee J P, Greenleaf J F, Riederer S J and Ehman R L 2000 MR imaging of shear waves generated by focused ultrasound Magn. Reson. Med. 43 111-5

Wu Z 2005 Shear wave interferometry and holography, an application of sonoelastography Doctoral Dissertation University of Rochester

Zadler B J, Le Rousseau J H, Scales J A and Smith M L 2004 Resonant ultrasound spectroscopy: theory and application Geophys. J. Int. 156 154-69

Zhai L, Madden J, Foo W C, Mouraviev V, Polascik T J, Palmeri ML and Nightingale K R 2010 Characterizing stiffness of human prostates using acoustic radiation force Ultrason. Imaging 32 201-13 